Results: The sample values gained with BÜHLMANN calibrators showed an excellent correlation with values gained with the WHO international standard for infliximab as calibrator. Passing-Bablok regression analysis revealed a slope of 0.96 and correlation coefficient $(\mathrm{R})$ of 0.99 . Bland-Altman analysis revealed a mean difference in the obtained values of less than five percent. Regarding spiking recovery analysis, all tests exhibit an excellent mean recovery of $101 \%(85-$ $114 \%$; a), 99\% (91-105\%; b); 101\% (95-107\%; c) and 94\% (88-100\%, d).

Conclusion: Current standardization of Quantum Blue $\AA$ Infliximab rapid test correlates very well with the WHO international standard for infliximab (NIBSC 16/170). Spiking recovery was highly comparable for ELISAs and the Quantum Blue ${ }^{\circledR}$ Infliximab assay. This rapid test represents a unique and modern analytical method, for fast time-to-result and simplicity of usage in a more patient near medical environment.

References:

[1] Westgard, James. (2008). Basic Method Validation.

Disclosure of Interests: None declared

DOI: 10.1136/annrheumdis-2020-eular.3322

\section{AB1111 PREDICTIVE VALUE OF FLUORESCENCE-OPTICAL IMAGING TECHNIQUE IN DETECTION OF PSORIATIC ARTHRITIS IN PSORIASIS PATIENTS}

M. Köhm ${ }^{1,2,3}$, S. Ohrndorf ${ }^{4}$, T. Rossmanith ${ }^{2,3}$, M. Backhaus ${ }^{5}$, G.

R. Burmester ${ }^{4}$, S. Wassenberg ${ }^{6}$, B. Köhler ${ }^{6}$, H. Burkhardt ${ }^{1,2,3}$, F. Behrens ${ }^{1,2,3}$. ${ }^{1}$ Goethe-University Frankfurt, Rheumatology Department, Frankfurt, Germany; ${ }^{2}$ Fraunhofer Cluster of Excellence Immun-Mediated Disease CIMD, Frankfurt, Germany; ${ }^{3}$ Fraunhofer IME Translational Medicine and Pharmacology, Frankfurt, Germany; ${ }^{4}$ Universitätsmedizin Charité, Berlin, Germany; ${ }^{5}$ Park-Klinik Weissensee, Berlin, Germany; ${ }^{6}$ Rheumazentrum Ratingen, Ratingen, Germany

Background: Psoriasis (Pso) is one of the most common chronic inflammatory skin diseases in Europe. Psoriatic arthritis (PSA) is closely associated to Pso whereas the skin manifestation appears usually years before PsA-related symptoms emerge. Up to $30 \%$ of Pso patients develop PsA, but there is no clear correlation between disease duration of Pso and PsA development. Therefore, biomarkers for its early detection are of major importance. In early PsA, changes in synovial vascularisation combined with increased expression of proangiogenic factors appear first. Therefore, imaging biomarkers for detection of changes in vascularisation might be useful for early detection of musculoskeletal disease. Fluorescence-optical imaging ( $\mathrm{FOI}$ ) is a new method to detect changes in microvascularisation of the hands.

Objectives: To determine the number of positive PsA diagnosis within a 24 month follow-up period in $\mathrm{PsO}$ only patients with subclinical MSK-inflammation detected in FOI.

Methods: Sensitivity of FOI for detection of subclinical signs of musculoskeletal inflammation as biomarker for early PsA was observed in a prospective, multicentre study (XCITING) including patients with dermatological confirmed skin psoriasis. 411 patients were included from dermatology care units across Germany without diagnosis of PsA but potential risk factors for its development (nail psoriasis and/or joint pain or swelling within the last 6 months). Clinical examination (CE; swollen (66) and tender (68) joint count, enthesitis, dactylitis assessment) and standardised ultrasound (US) assessment was performed by a qualified rheumatologist to assess musculoskeletal inflammation. FOI was performed additionally. Data was analysed in focus on increased vascularisation of musculoskeletal structures as inflammatory markers. In case of discrepant results (positive FOI and negative CE and US), MRI was performed to prove the findings. In case of MRI negativity, a follow-up period of 24-months was performed including FOI, CE, US and MRI assessment.

Results: 83 of the 411 patients of the cohort were negative in all assessments (Pso only), 136 of the 411 patients were classified as PsA by rheumatologic assessments. 119 patients showed subclinical signs of musculoskeletal inflammation in the central reading of $\mathrm{FOI}$, whereas CE and US were negative. In $37,5 \%$ of those patients, subclinical inflammation was confirmed by MRI assessment. 22 patients of the cohort without MRI positivity were willing to be followed up until month 24. $5(7.5 \%)$ patients developed a clinical PsA until month 24 whereas $7(10.5 \%)$ patients converted to be FOI negative. In 5 patients an additional MRI examination was performed in which one patient showed positive signs for inflammation.

Conclusion: $\mathrm{FO}$ is an innovative method for measurement of changes in microvascularisation in the hands. 6/22 patients initial only positive in FOI (no clinical signs for PsA, negative US, negative MRI) developed either clinical evident PsA ( $n=5)$ or new inflammation in MRI $(n=1)$ during follow-up of 24 months. Therefore, FOI positive signals in $\mathrm{PsO}$ patients increase the probability for PsA development.

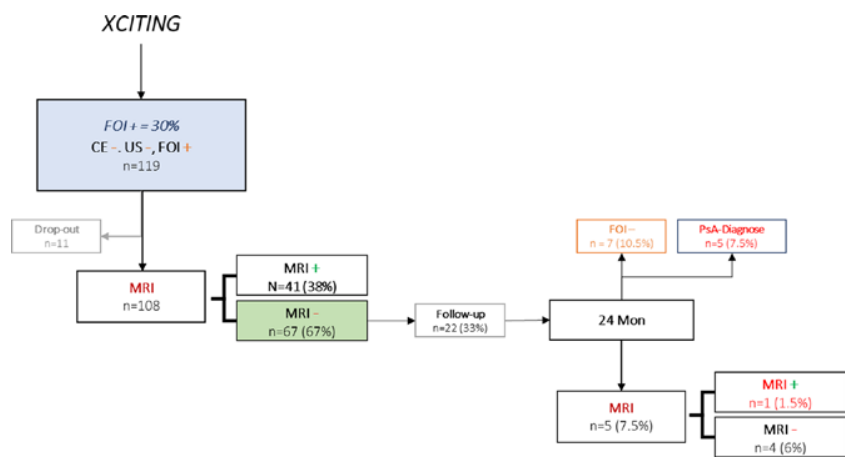

Figure 1. Flow Chart of the Follow-up period of the XCITING study.

Disclosure of Interests: Michaela Köhm Grant/research support from: Pfizer Janssen, BMS, LEO, Consultant of: BMS, Pfizer, Speakers bureau: Pfizer, BMS, Janssen, Novartis, Sarah Ohrndorf: None declared, Tanja Rossmanith Grant/ research support from: Janssen, BMS, LEO, Pfizer, Marina Backhaus Grant/ research support from: Pfizer, Gerd Rüdiger Burmester Consultant of: AbbVie Inc, Eli Lilly, Gilead, Janssen, Merck, Roche, Pfizer, and UCB Pharma, Speakers bureau: AbbVie Inc, Eli Lilly, Gilead, Janssen, Merck, Roche, Pfizer, and UCB Pharma, Siegfried Wassenberg: None declared, Benjamin Köhler Grant/ research support from: Pfizer, Harald Burkhardt Grant/research support from: Pfizer, Roche, Abbvie, Consultant of: Sanofi, Pfizer, Roche, Abbvie, Boehringer Ingelheim, UCB, Eli Lilly, Chugai, Bristol Myer Scripps, Janssen, and Novartis, Speakers bureau: Sanofi, Pfizer, Roche, Abbvie, Boehringer Ingelheim, UCB, Eli Lilly, Chugai, Bristol Myer Scripps, Janssen, and Novartis, Frank Behrens Grant/ research support from: Pfizer, Janssen, Chugai, Celgene, Lilly and Roche, Consultant of: Pfizer, AbbVie, Sanofi, Lilly, Novartis, Genzyme, Boehringer, Janssen, MSD, Celgene, Roche and Chugai

DOI: 10.1136/annrheumdis-2020-eular.5300

\section{AB1112 THE DIAGNOSTIC OF THE OSTEOARTHRITIS OF THE HANDS BY CONVENTIONAL RADIOGRAPHY}

D. Kudinsky ${ }^{1}$, L. Alekseeva ${ }^{2}$, A. Smirnov ${ }^{1}$, A. Volkov ${ }^{1}$, O. Alekseeva ${ }^{1}$, E. Taskina ${ }^{2}$, A. Sukhinina ${ }^{1} .{ }^{1}$ Moscow, Diagnostic Department, Moscow, Russian Federation; ${ }^{2}$ Moscow, Clinical Department, Moscow, Russian Federation

Background: The most severe phenotype of osteoarthritis (OA) is currently considered to be an inflammatory or erosive phenotype (EOA). There is currently no reliable $x$-ray picture of this disease in the literature, and the question of whether it is an independent form of $\mathrm{OA}$, a natural more pronounced stage of progression, or a separate nosology is debated in the literature.

Objectives: To identify the localization, frequency, and severity of pain and radiological symptoms in patients with EOA and non-erosive (NOA) disease in the interphalangeal (DIP and PIP) and metacarpal (MCP) joints of the hands.

Methods: 64 women with diagnosis of OA of the hand (HOA) joints according to the ACR criteria were included into study after signing the informed consent form. Mean age was $65.28 \pm 6.82$ years (48-77), mean BMl $27,7 \pm 4,4 \mathrm{~kg} / \mathrm{m}^{2}$, mean disease duration $12 \pm 8,1$ years. Individual patient's medical record included relevant anthropometric data, records from case history and clinical examination, AUSCAN scores, patient's articular status. Instrumental diagnostic methods included plain radiography of the hand joints in an anterior-posterior projection. The images were described in accordance with the Kellgren\&Lawrence (K\&L) system.

When evaluating radiographs of 64 patients with $\mathrm{HOA}$, the most common was stage II (49\%) according to $\mathrm{K} \& \mathrm{~L}$, and the most common symptoms in distal (DIP), proximal (PIP) interphalangeal and MCP were joint space narrowing (JSN) $(100 \%, 100 \%$, and $95 \%$, respectively) and osteophytes (OP) $(88 \%, 70 \%$, and $45 \%$, respectively). Subchondral osteosclerosis (SO) $(5 \%)$, erosions $(8 \%)$, and subluxations (3\%) in MCP, as well as subluxation in PIP $(6 \%)$ were less common. Statistica 10.0 was used for statistical analysis.

23 patients had EOA, 37 had NOA. Depending on the presence of erosions in interphalangeal joints patients were divided into 2 groups comparable in terms of age, age of OA onset and duration of disease (the average age of patients with EOA interphalangeal joints was $68+6.15$ years, and mean disease duration $18,34+7.11$ years; in the group without erosive changes in the average age amounted to $65,13 \pm 5.43$ years, mean disease duration of $16.56 \pm 8.4$ years).

Results: EOA DIP and PIP was detected in $15(23 \%)$ with radiological changes corresponding to stages III-IV of HOA and in 8 people (12\%) with stage II on 